\title{
Supernova Relic Neutrino Search and Atmospheric Neutrino Neutral-Current Quasi-Elastic Interactions Measurement in Super-Kamiokande
}

\author{
WAN, Linyan* for the Super-Kamiokande collaboration \\ Department of Engineering Physics, Tsinghua University \\ E-mail: wanly13@mails.tsinghua.edu.cn
}

Supernova relic neutrinos (SRN), also called the diffused supernova neutrino backgrounds (DSNB), are emitted from core-collapse supernovae throughout the universe. Super-KamiokandeIV tags inverse beta interactions by neutrons captured on hydrogen using improved electronics and triggering. Recently, the use of vertex reconstruction of the $2.2 \mathrm{MeV}$ photons emitted by the neutron captures and a neural network improved the discrimination against backgrounds and significantly increased the efficiency. Neutral current quasi-elastic (NCQE) scattering of atmospheric neutrinos on ${ }^{16} \mathrm{O}$ in water-Cherenkov detectors forms an important remaining background in SRN detection. Using neutron tagging technique, the Super-Kamiokande measurement of the NCQE cross section of atmospheric neutrinos will be presented and compared against theoretical predictions.

ICHEP 2018, International Conference on High Energy Physics

4-11 July 2018

Seoul, South Korea

${ }^{*}$ Speaker. 


\section{Introduction}

Neutral current quasi-elastic interaction (NCQE) from atmospheric neutrinos with nuclei is found to be one of the major interaction channels for neutrinos with several hundred $\mathrm{MeV}$ energy [1]. The interaction processes can be written as

$$
\begin{aligned}
& v+{ }^{A} \mathrm{X} \rightarrow v+{ }^{A-1} \mathrm{X}+n+\gamma^{\prime} s, \\
& v+{ }^{A} \mathrm{X} \rightarrow v+{ }^{A-1} \mathrm{X}-1+p+\gamma^{\prime} s,
\end{aligned}
$$

in which the incident neutrino knocks out a nucleon from the nucleus and the residual nucleus is likely to produce de-excitation $\gamma$ 's.

Super-Kamiokande (SK) is a water Cherenkov neutrino experiment located in Kamioka, Japan [3]. The detector is a cylindrical water tank loaded with 50 kiloton of pure water. At SK, although the majority of NCQE $\gamma$ 's can be distinguished by their larger and more smeared Cherenkov angles from electrons, a considerable amount of remnant are still mis-reconstructed as electrons. Among the emitted nucleons, neutrons will be captured on hydrogen, releasing a $2.2 \mathrm{MeV}$ photon that can be tagged. Due to the spacial and time correlation, the de-excitation $\gamma$ ray and the emitted neutron of an NCQE event can mimic an inverse beta decay (IBD, $\bar{v}_{e}+p \rightarrow n+e^{+}$) event, thereby contaminating the data sample of rare signal search, especially for supernova relic neutrinos [4].

A measurement from $\mathrm{T} 2 \mathrm{~K}$ neutrino beam reports a result in agreement with the theoretical prediction [5], but the average cross-section on oxygen from atmospheric neutrinos has not been studied before. A precise knowledge on the NCQE from atmospheric neutrinos is highly motivated in both the aspect of nuclear physics and the rare signal search in future water Cherenkov detectors such as SK-Gd [6] and Hyper-K [7].

\section{Simulation}

In this analysis, we use the HHKM model as the input profile of atmospheric neutrinos [8, 9]. The Monte-Carlo simulation of atmospheric neutrino events is performed in two stages, the generator level to model the neutrino interaction, and the detector simulation level to track the nucleons and $\gamma$ 's. The neutrino interactions at SK is modelled by the generator NEUT [10]. The NCQE cross-section on oxygen in NEUT is simulated by an oxygen spectral function model [11] with the BBBA05 form factor [12], taking into consideration the Pauli blocking effect at $p_{F}=225$ $\mathrm{MeV} / c$. The neutrino knocking out a neutron from the oxygen nucleus, leaving a hole in $1 p_{1 / 2}$, $1 p_{3 / 2}$ or $1 s_{1 / 2}$ state in the simple shell model. Among them, the states with the largest branching ratios will emit a $\sim 6 \mathrm{MeV} \gamma$.

For the $\gamma$ 's and nucleons, a GEANT3 [13] based SK detector simulation package, named SKDETSIM, is employed at SK. It tracks detector response to the emitted $\gamma$ 's and nucleons, as well as the secondary processes, in which primary nucleons with higher energy further collide on other oxygen nuclei, knocking out additional nucleons and producing more $\gamma$ 's.

\section{Data Analysis}

SK has undergone four data taking phases of SK-I/II/III/IV starting from 1996. This analysis 
uses 2,778 days of SK-IV data in a fiducial volume of 22.5 kiloton. To avoid the overlap with the dominant reactor neutrino background, the analyzed energy range is determined as $[8,30] \mathrm{MeV}$.

During the data reduction, first reduction removes nonphysical triggers as well as incoming muons with an efficiency $>99 \%$ [14]. After that, the spallation cut is applied to reject the spallation products from the cosmic ray muons with a longer life-time than the muon veto time window [15] at an efficiency dependent on the energy from $51 \%$ to $90 \%$. Further reductions aim to remove backgrounds with a distinct signature such as incoming $\gamma$ 's from PMT surfaces, solar neutrinos, multi-rings which are likely come from atmospheric charged currents, and muons and pions [16]. The Cherenkov angle of an electron above the threshold peaks at $42^{\circ}$, while for low energy $\gamma$ 's it is more uniform and smeared, usually reconstructed above $50^{\circ}$, as shown in the left panel of Fig. 1.

Neutron capture $\gamma$ 's are recorded in the SK data flow via a special trigger scheme called SHEAFT [2]. The true neutrons are tagged from the data by a technique called neutron tagging [16]. Recently an upgraded neutron tagging algorithm taking into consideration the reconstruction of neutron capture vertex using both the unbiased BONSAI algorithm [17] and a brute-force fitter has been developed, enhancing greatly the discrimination power between neutron capture signals and accidental coincidence backgrounds.
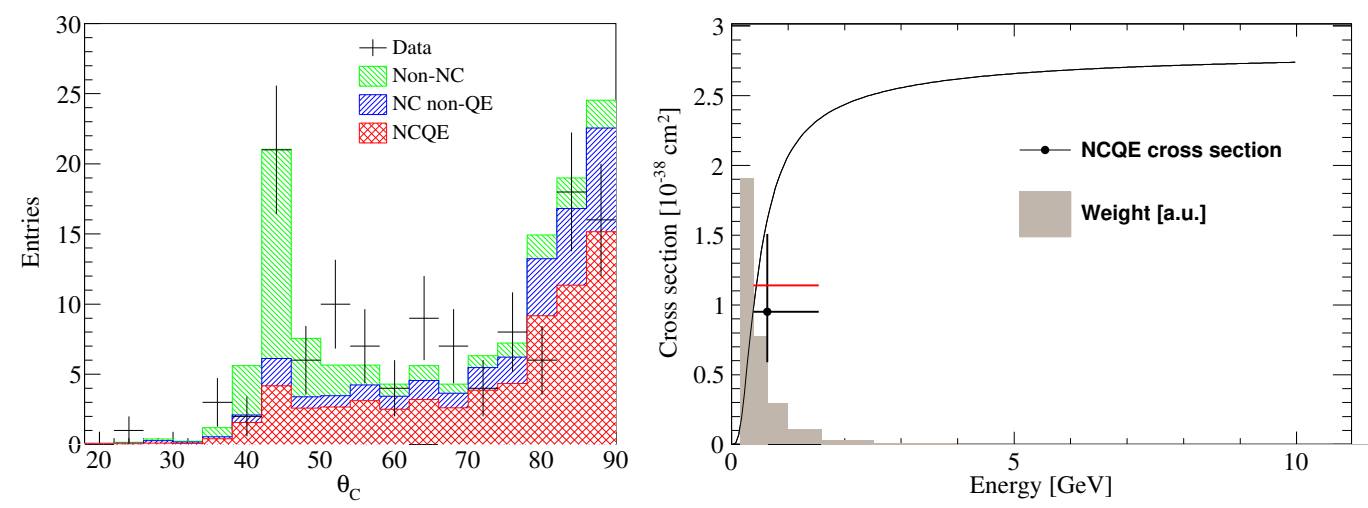

Figure 1: Left: Cherenkov angle distribution in data (black points) and MC expectation (colored histograms) after all other cuts and with neutron tagging. Right: the measured (black point) and the expected (red line) average cross-section.

The measured cross-section is calculated by

$$
<\sigma_{\mathrm{NCQE}}^{\text {observed }}>=\frac{N_{\text {tot }}^{\mathrm{obs}}-N_{\mathrm{acc}}^{\mathrm{obs}}-N_{\mathrm{others}}^{\mathrm{obs}}-N_{\mathrm{NCothers}}^{\mathrm{exp}}}{N_{\mathrm{NCQE}}^{\mathrm{exp}}} \times<\sigma_{\mathrm{NCQE}}^{\text {theory }}>,
$$

where $N_{\text {tot }}^{\text {obs }}$ refers to the observed number of events, $N_{\text {acc }}^{\text {obs }}$ refers to the accidental background without a true neutron, and $N_{\text {others }}^{\text {obs }}$ refers to the non-NC backgrounds including spallation products, reactor antineutrinos, and atmospheric neutrino $\mathrm{CC}$ interactions. The theoretical prediction $<\sigma_{\mathrm{NCQE}}^{\text {theory }}>$ is integrated between $160 \mathrm{MeV}$ and $10 \mathrm{GeV}$, above which the atmospheric neutrino flux is decreasing rapidly, and below which the NCQE cross-section is very small and negligible. The atmospheric neutrino spectrum averaged NCQE cross-section on oxygen is measured to be $(0.95 \pm 0.12 \text { (stat. })_{-0.32}^{+0.49}$ (sys. $\left.)\right) \times 10^{-38} \mathrm{~cm}^{2}$, as shown in the right panel in Fig. 1 . The systematics are mainly contributed from the atmospheric neutrino profile, the simulation for relevant $\mathrm{NC}$ process, and the neutron tagging. 


\section{Conclusion}

We report here the first measurement of NCQE cross-section from atmospheric neutrinos on oxygen. NCQE events are selected by the coincidence of nuclear de-excitation gamma and neutron capture signal on hydrogen using the neutron tagging technique. The NCQE cross section from atmospheric neutrinos is measured to be $(0.95 \pm 0.12 \text { (stat. })_{-0.32}^{+0.49}$ (sys. $\left.)\right) \times 10^{-38} \mathrm{~cm}^{2}$, consistent with the theoretical prediction of $1.14 \times 10^{-38} \mathrm{~cm}^{2}$. This result improves the estimation of NCQE component in low energy rare signal detection, especially in the search of supernova relic neutrinos in future water Cherenkov experiments, such as SK-Gd and Hyper-K.

\section{References}

[1] A. M. Ankowski, O. Benhar, T. Mori, R. Yamaguchi and M. Sakuda, Phys. Rev. Lett. 108, 052505 (2012) doi:10.1103/PhysRevLett.108.052505 [arXiv:1110.0679 [nucl-th]].

[2] H. Watanabe et al. [Super-Kamiokande Collaboration], Astropart. Phys. 31, 320 (2009) doi:10.1016/j.astropartphys.2009.03.002 [arXiv:0811.0735 [hep-ex]].

[3] Y. Fukuda et al. [Super-Kamiokande Collaboration], Nucl. Instrum. Meth. A 501, 418 (2003). doi:10.1016/S0168-9002(03)00425-X

[4] A. Gando et al. [KamLAND Collaboration], Astrophys. J. 745, 193 (2012) doi:10.1088/0004-637X/745/2/193 [arXiv:1105.3516 [astro-ph.HE]].

[5] K. Abe et al. [T2K Collaboration], Phys. Rev. D 90, no. 7, 072012 (2014) doi:10.1103/PhysRevD.90.072012 [arXiv:1403.3140 [hep-ex]].

[6] H. Sekiya, J. Phys. Conf. Ser. 888, no. 1, 012041 (2017). doi:10.1088/1742-6596/888/1/012041

[7] K. Abe et al., arXiv:1109.3262 [hep-ex].

[8] M. Honda, T. Kajita, K. Kasahara, S. Midorikawa and T. Sanuki, Phys. Rev. D 75, 043006 (2007) doi:10.1103/PhysRevD.75.043006 [astro-ph/0611418].

[9] M. Honda, T. Kajita, K. Kasahara and S. Midorikawa, Phys. Rev. D 83, 123001 (2011) doi:10.1103/PhysRevD.83.123001 [arXiv:1102.2688 [astro-ph.HE]].

[10] Hayato, Yoshinari Acta Phys. Polon. B40, 2477-2489 (2009)

[11] O. Benhar, N. Farina, H. Nakamura, M. Sakuda and R. Seki, Phys. Rev. D 72, 053005 (2005) doi:10.1103/PhysRevD.72.053005 [hep-ph/0506116].

[12] R. Bradford, A. Bodek, H. S. Budd and J. Arrington, Nucl. Phys. Proc. Suppl. 159, 127 (2006) doi:10.1016/j.nuclphysbps.2006.08.028 [hep-ex/0602017].

[13] R. Brun, F. Bruyant, M. Maire, A. C. McPherson and P. Zanarini, CERN-DD-EE-84-1.

[14] K. Abe et al. [Super-Kamiokande Collaboration], Phys. Rev. D 94, no. 5, 052010 (2016) doi:10.1103/PhysRevD.94.052010 [arXiv:1606.07538 [hep-ex]].

[15] K. Bays et al. [Super-Kamiokande Collaboration], Phys. Rev. D 85, 052007 (2012) doi:10.1103/PhysRevD.85.052007 [arXiv:1111.5031 [hep-ex]].

[16] H. Zhang et al. [Super-Kamiokande Collaboration], Astropart. Phys. 60, 41 (2015) doi:10.1016/j.astropartphys.2014.05.004 [arXiv:1311.3738 [hep-ex]].

[17] M. Smy [Super-Kamiokande Collaboration], INSPIRE-1371616. 